\title{
Choosing a nursing career: Building an indigenous nursing workforce
}

\author{
Linda Deravin ${ }^{* 1}$, Karen Francis ${ }^{2}$, Judith Anderson ${ }^{1}$ \\ ${ }^{1}$ School of Nursing Midwifery and Indigenous Health, Charles Sturt University, New South Wales, Australia \\ ${ }^{2}$ School of Nursing Midwifery and Nutrition, Janes Cook University, Queensland, Australia
}

Received: June 21, 2017

Accepted: September 7, $2017 \quad$ Online Published: September 18, 2017

DOI: $10.5430 /$ jha.v6n5p27

URL: https://doi.org/10.5430/jha.v6n5p27

\begin{abstract}
Introduction: This paper provides an overview of the impact of government policy in supporting the growth of an Indigenous nursing workforce in New South Wales and Australia.

Methods: Publically available nursing workforce performance reports along with current literature were reviewed to provide a perspective on the current situation.

Results and discussion: The National partnership agreement on closing the gap in Indigenous health outcomes indicated that to improve Indigenous health outcomes, significant investment is required with particular reference to increasing an Indigenous workforce. As nurses comprise the majority of the health workforce a number of strategies and initiatives have been put in place to support this agreement. Even though there has been commitment through government policy and funding initiatives it is questionable if this is having any real impact on growing an Indigenous nursing workforce.

Conclusions: Recruitment strategies that will increase the number of Indigenous nurses within the health workforce requires a multilevel approach. Despite efforts to increase Indigenous nursing workforce numbers, there has been limited impact to any real successful gains since 2008. Building and growing an Indigenous nursing workforce that will support the "Closing the Gap" initiative will require significant continuing effort.
\end{abstract}

Key Words: Indigenous, Nursing, Workforce, Education pathways, Recruitment and retention, Health inequity

\section{INTRODUCTION}

Indigenous Australians represent $2.5 \%$ of the Australian population yet have a lower life expectancy, higher disease burden and poorer health outcomes compared to non- Indigenous Australians. ${ }^{[1]}$ A key strategy to improve the health and wellbeing of this population group was to encourage more Indigenous people to enter the health profession. This paper provides a focus on the current Indigenous nursing workforce as one example of a health profession employed within the health sector. A brief discussion on the impact of policy in supporting the growth of the nursing workforce and an outline of the available resources that support Indigenous people to enter the nursing profession will also be discussed. As a comparative view, both national and state data will be compared with a particular focus on one state's experience being New South Wales (NSW), to demonstrate outcomes to date. Recommendations for future opportunities to support and develop an Indigenous nursing workforce will also be indicated.

In 2008, the Australian government developed the "National

\footnotetext{
*Correspondence: Linda Deravin; Email: lderavin@csu.edu.au; Address: School of Nursing, Midwifery and Indigenous Health, Bathurst, NSW, 2795, Australia.
}

Published by Sciedu Press 
partnership agreement on closing the gap in Indigenous health outcomes", ${ }^{[2]}$ as a way to address this inequity by working in partnership with all states and territories to improve the health of Indigenous people. One of the components within this agreement indicated that to improve Indigenous health outcomes, significant investment was required with particular reference to building an Indigenous workforce. The "National health workforce innovation and reform strategic framework for action 2011-2015" developed by Health Workforce Australia, ${ }^{[3]}$ a now defunct entity, was established to co-ordinate the reform. Within this plan a commitment to reducing the gap between the Indigenous and non-Indigenous workforce within the next 10 years is articulated. ${ }^{[3]}$ The nursing profession make up the majority of the health workforce, therefore the need to increase Indigenous nursing workforce numbers is paramount in order to support health programs and policy to address this health inequity.

\section{METHOD}

In order to gain an understanding of the current Indigenous nursing workforce, publically available performance report documents were sourced from various government websites. As a way to expand beyond just an interpretation of the data, current literature was also examined (within the last 6 years) using key search terms such as nursing, Indigenous, workforce, and education which reported on progress or provided commentary on this issue. Only 7 articles met the inclusion criteria within the literature demonstrating that little is known about this issue.

\section{RESULTS AND DISCUSSION}

\subsection{Indigenous nursing workforce - current status}

Nurses are at the forefront of health care to deliver and effect change that makes an impact on patient outcomes. Components that must be considered to build an Indigenous nursing workforce include education and employment strategies to fill this void. ${ }^{[4]}$ As demonstrated in Table 1, nurses that identify as being of Indigenous descent across the entire nation rose from $0.6 \%$ in 2008 to $1.1 \%$ in 2015 , indicating a $99 \%$ increase. However in real terms the actual numbers only increased by $1,589(1,598$ to 3,187$)$. In a nation where there are over 307,000 nurses employed within the health system this increase was minor. The most significant increase occurred in NSW where the Indigenous nursing workforce grew by 590 from 2008 to 2015 (see Table 1). Even though there has been commitment through government policy and funding initiatives at both the federal and state level it is questionable that these initiatives are having any real impact on growing an Indigenous nursing workforce. If the Indigenous population are $2.5 \%$ of the national population, it is reasonable to assume that numbers within the existing nursing workforce should be similar. In 2015 it was reported that there were 307,104 nurses in Australia. To reach 2.5\% there would need to be 7,677 Indigenous nurses. ${ }^{[5]}$ The current growth rate of $14.12 \%$ per year indicates that it will possibly be another 26 years before the percentage of Indigenous nurses mirrors the percentage of the general population that are Indigenous. The evidence demonstrates that growth has been slow.

Table 1. Comparison of Indigenous nursing workforce to total nursing workforce in NSW and Australia (2008-2015- latest data available)

\begin{tabular}{|c|c|c|c|c|c|c|c|}
\hline & 2008 & 2009 & 2011 & 2012 & 2013 & 2014 & 2015 \\
\hline Number of RN and EN's who identify as Indigenous in NSW & 598 & 650 & 862 & 865 & 994 & 1,184 & 1,188 \\
\hline$\%$ of nursing workforce NSW & 0.8 & 0.8 & 1.1 & 1.1 & 1.2 & 1.4 & 1.4 \\
\hline Number of RN and EN's who identify as Indigenous in Australia & 1,598 & 1,605 & 2,246 & 2,301 & 2,601 & 3,036 & 3,187 \\
\hline$\%$ of nursing workforce Australia & 0.6 & 0.6 & 0.8 & 0.8 & 0.9 & 1.0 & 1.1 \\
\hline
\end{tabular}

Note. Data not available for $2010 \cdot{ }^{[5-11]} \mathrm{RN}$ denotes registered nurse and EN denotes enrolled nurse, these designations are those nurses who are credentialed within Australia

Government policy documents celebrate that their efforts to increase Indigenous nurses within the health workforce are successful with numbers almost doubling at both the state and federal level from 2008 to 2015..$^{[5,12]}$ However, actual numbers remain low and with the percentage of Indigenous nurses being reported at $1.1 \%$ of the entire nursing workforce nationally, there has been minimal impact to the numbers of Indigenous nurses. West et al. ${ }^{[4]}$ agreed that although many strategies to address the Indigenous repre- sentation in nursing have been instigated these in fact have shown little improvement overall. With a $75 \%$ increase in Indigenous nursing numbers in NSW from 2008 to 2015 and an increase nationally of $83 \%$, statistically this would semm that progress is going well however in real terms with actual numbers increasing slowly as indicated previously it is obvious that more needs to be done to support Indigenous people to choose nursing as a career within the health workforce. Stuart and Nielsen ${ }^{[13]}$ affirmed the need for increasing In- 
digenous nurses to help bridge the gap and provide culturally appropriate care.

\subsection{Pathways into nursing}

In order to support the growth in numbers of Indigenous nurses, consideration should be given to available pathways to enter the nursing profession. ${ }^{[4,14]}$ There are a number of pathways available to enter nursing at either the vocational education and training, or university level as places of tertiary education. Options for study currently include: assistant in nursing, enrolled nurse and registered nurse. Depending on the qualification, time spent undertaking study in order to be qualified for one of these positions ranges from 12 months to obtain a certificate level 3 to a minimum of 3 years full time study to become a registered nurse. Competition by education providers to entice Indigenous people to join the nursing workforce is fierce, particularly in light of other health roles that are available.

An alternate health professional role for those who may potentially consider nursing is the Indigenous health worker role. This role differs from state to state, but was commonly designed to provide culturally safe care to Indigenous peoples, supporting their visits to health professionals and providing primary health care. These workers are employed by a variety of organisations and are regulated by the Aboriginal and Torres Strait Islander Health Practice Board of Australia. ${ }^{[15]}$ Qualifying as an Indigenous Health Worker for example has an entry level qualification of a certificate level 3 which can be completed in 6 weeks depending on where there initial qualification is obtained.

These and many other health professional roles have a place in the delivery of health care to bridge the gap in health inequity for Indigenous people yet how do we continue to grow and expand the nursing workforce when such competition exists?

\subsection{Supporting success}

Many students when entering tertiary education, regardless of cultural background, find the financial impost a major barrier to the successful completion of their studies. ${ }^{[4]}$ There are a number of financial scholarships available for Indigenous people to access as a way to manage the financial hardship of attending university. These scholarships are provided from a range of sources including government departments such as health, university scholarships and private organisations. Additional support is also provided in mentoring programs and cultural support groups based within the tertiary education sector. Even with these programs in place, successful completion rates of Indigenous people undertaking tertiary education remain low. ${ }^{[16]}$

Published by Sciedu Press
West et al. ${ }^{[4]}$ recommended ways to move forward in addressing these issues require a combined approach. Some of the suggested strategies include incorporating an Indigenous pedagogy within undergraduate nursing programs to educate non Indigenous people about traditional culture and attitudes toward health systems which are not culturally sensitive. ${ }^{[17]}$ Another strategy suggests that more Indigenous nurses should be employed within universities so that they may act as supports to Indigenous nursing students and provide good role models. Ideally developing a critical mass of Indigenous students so that cultural support may be provided by peers is another important aspect to consider ${ }^{[4]}$ yet obtaining this critical mass is the challenge currently faced.

\section{Conclusions}

Despite efforts to increase nursing workforce numbers, there has been limited impact to any real successful gains in the nursing workforce since 2008. In considering recruitment strategies that will build and increase the number of nurses within the Indigenous health workforce, a multilevel approach involving government, the tertiary education sector and employers is required. ${ }^{[8,18]}$ One of the considerations to encourage more people to enter the nursing profession is mapping of existing pathways of other health worker roles such as the Aboriginal and Torres Strait Islander worker to nursing programs that recognises the skills and qualifications attained. Even with the available supports for Indigenous people entering tertiary study, retention and completion rates remain low. ${ }^{[17]}$ It is essential that culturally appropriate support strategies are provided within all education sectors to address this disparaging anomaly.

Indigenous people through the support of their communities, should be encouraged to enter into the nursing profession. Even though in some cultures entering the nursing profession may not be seen as an attractive career, within Australia the profession is highly valued. There are limited studies that have investigated Indigenous representation in the Australian nursing workforce despite the profession recognising that this group are necessary if the needs of Indigenous Australians are to be met. ${ }^{[16,17,19]}$ Indigenous people who have successfully achieved a higher level of education by becoming a nurse should be celebrated and promoted as role models for Indigenous people. ${ }^{[16]}$ Opportunities to promote nursing as a career option and participate in decision making processes that affect Indigenous people regarding health policy should be created and encouraged. ${ }^{[19]}$ Building and growing an Indigenous nursing workforce will support the "Closing the Gap" initiative yet without significant efforts to achieve this, the initiative will continue to struggle in achieving its outcomes. 
CONFLiCTS OF INTEREST Disclosure

\section{REFERENCES}

[1] COAG Reform Council. National Indigenous reform agreement: Performance report for 2009-10. Sydney: COAG Reform Council; 2011.

[2] Council of Australian Governments. National partnership agreement on closing the gap in Indigenous health outcomes. In: Relations F, editor.: Australian Government; 2008.

[3] Health Workforce Australia. National health workforce innovation and reform strategic framework for action 2011-2015. Adelaide. 2011.

[4] West R, Usher K, Foster K. Increased numbers of Australian Indigenous nurses would make a significant contribution to 'closing the gap' in Indigenous health: What is getting in the way? Contemporary Nurse. 2010; 36(1-2): 121-30. PMid: 21254828. https://doi.org/10.5172/conu.2010.36.1-2.121

[5] Australian Institute of Health and Welfare. Nursing and midwifery workforce 2015- Nurses and midwives, overview tables 1-25. Canberra: AIHW; 2016.

[6] Australian Institute of Health and Welfare. Nursing and midwifery work force 2011-Registered and enrolled nurses, demographic overview, tables 1-12. Canberra: AIHW; 2012.

[7] Australian Institute of Health and Welfare. Nursing and midwifery workforce 2012. Canberra: AIHW; 2013.

[8] Australian Institute of Health and Welfare. Nursing and midwifery workforce 2013 supplemmentary tables. Canberra: AIHW; 2014.

[9] Australian Institute of Health and Welfare. Nursing and midwifery workforce 2014: Nurses and midwives, overview tables 1-25. Canberra: AIHW; 2015.

[10] Australian Institute of Health and Welfare. Nurses overview, tables 1-34, figures 1-4 for 20082010.
[11] Australian Institute of Health and Welfare. Nurses overview, tables 1-34, figures 1-4 for 2009. 2011.

[12] Health Workforce Australia. Australia's Health Workforce Series Aboriginal and Torres Strait Islander Health Workers/Practitioners in focus. In: Division HW, editor. Canberra: Department of Health; 2014.

[13] Stuart L, Nielsen AM. Two Aboriginal registered nurses show us why black nurses caring for black patients is good medicine. Contemporary Nurse. 2011; 37(1): 96-101.

[14] Deravin-Malone L. Creating pathways in nursing education to increase Indigenous nursing numbers. Australian Nursing and Midwifery Journal. 2016; 23(8): 37. PMid: 27132392.

[15] Health Workforce Australia. Australia's Health Workforce SeriesAboriginal and Torres Strait Islander health workers/Practitioners in focus. In: Division HW, editor. Canberra: Health Workforce Australia; 2014.

[16] Best O, Stuart L. An Aboriginal nurse-led working model for success in graduating Indigenous Australian nurses. Contemporary Nurse. 2014; 48(1): 59-66. PMid: 25410196. https://doi.org/10.108 $0 / 10376178.2014 .11081927$

[17] West R, Usher K, Buettner PG, et al. Indigenous Australians' participation in pre-registration tertiary nursing courses: A mixed methods study. Contemporary Nurse. 2013; 46(1): 123-34. PMid: 24716770. https://doi.org/10.5172/conu.2013.46.1.123

[18] Usher K. Indigenous higher degree research students making a difference to the Indigenous health agenda. Contemporary Nurse. 2011; 37(1): 102-6.

[19] Stuart L, Gorman D. The experiences of Indigenous health workers enrolled in a Bachelor of Nursing at a regional Australian university. An International Journal of Indigenous Peoples. 2015; 11(1): 29-44. https://doi.org/10.1177/117718011501100103 